\title{
SPATIAL LIGHT MODULATORS
}

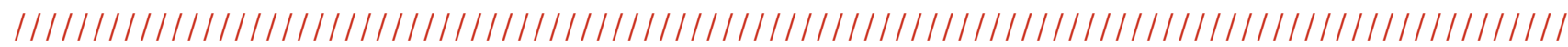

\section{Aurélie JULLIEN}

Institut de Physique de Nice, Valbonne, France - aurelie.jullien@inphyni.cnrs.fr

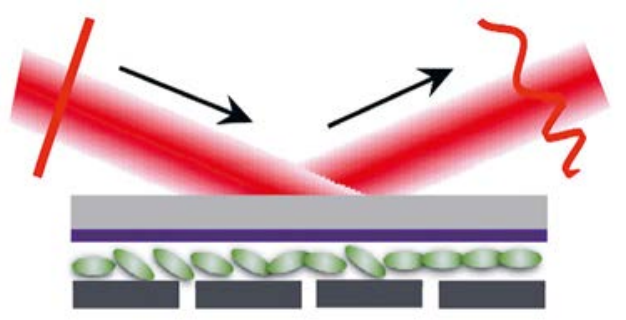

Spatial Light Modulators (SLMs) are quasiplanar devices, allowing for the modulation of the amplitude, phase and polarization, or a combination of these parameters of an incident light beam according to the two spatial dimensions of the modulator. SLMs are employed in many different fields and are the subject of continuous technological development.

https://doi.org/10.1051/photon/202010159

This is an Open Access article distributed under the terms of the Creative Commons Attribution License (http://creativecommons.org/licenses/by/4.0), which permits unrestricted use, distribution, and reproduction in any medium, provided the original work is properly cited.

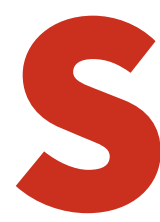
patial light modulation is a well-established optical technology with a wide range of applications. Spatial light modulators (SLMs) are two-dimensional objects, enabling to modulate, at any point of the SLM surface, through a local change of the optical path, the intensity, phase or polarization of an incident light beam. They are usually organized into categories according to (i) their use in reflection or transmission, (ii) the modulated optical parameter(s): amplitude, phase, polarization, and (iii) the type of the driving signal: electrical or optical.

Although spatial light modulation has been made possible through a plethora of technologies, among them mechanically or thermally deformable mirrors, digital micro-mirror device (DMD), magneto-optic devices or acoustic-optic Bragg cells,

\section{... when every photon counts}
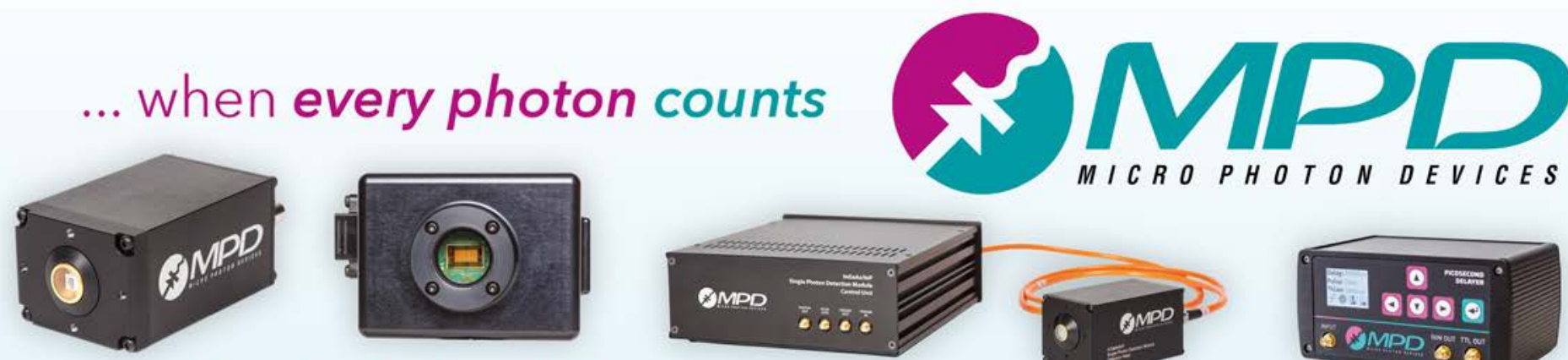

visible and infrared single-photon counters

single-photon counting and timing SPAD cameras

picosecond delayers and QRN generators

fast-gated single-photon counters
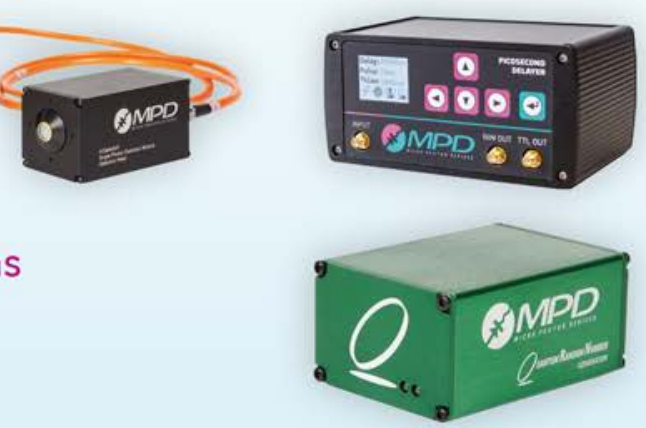

MICRO PHOTON DEVICES S.r.I. Tel. +39 0471051212 www. micro-photon-devices.com 

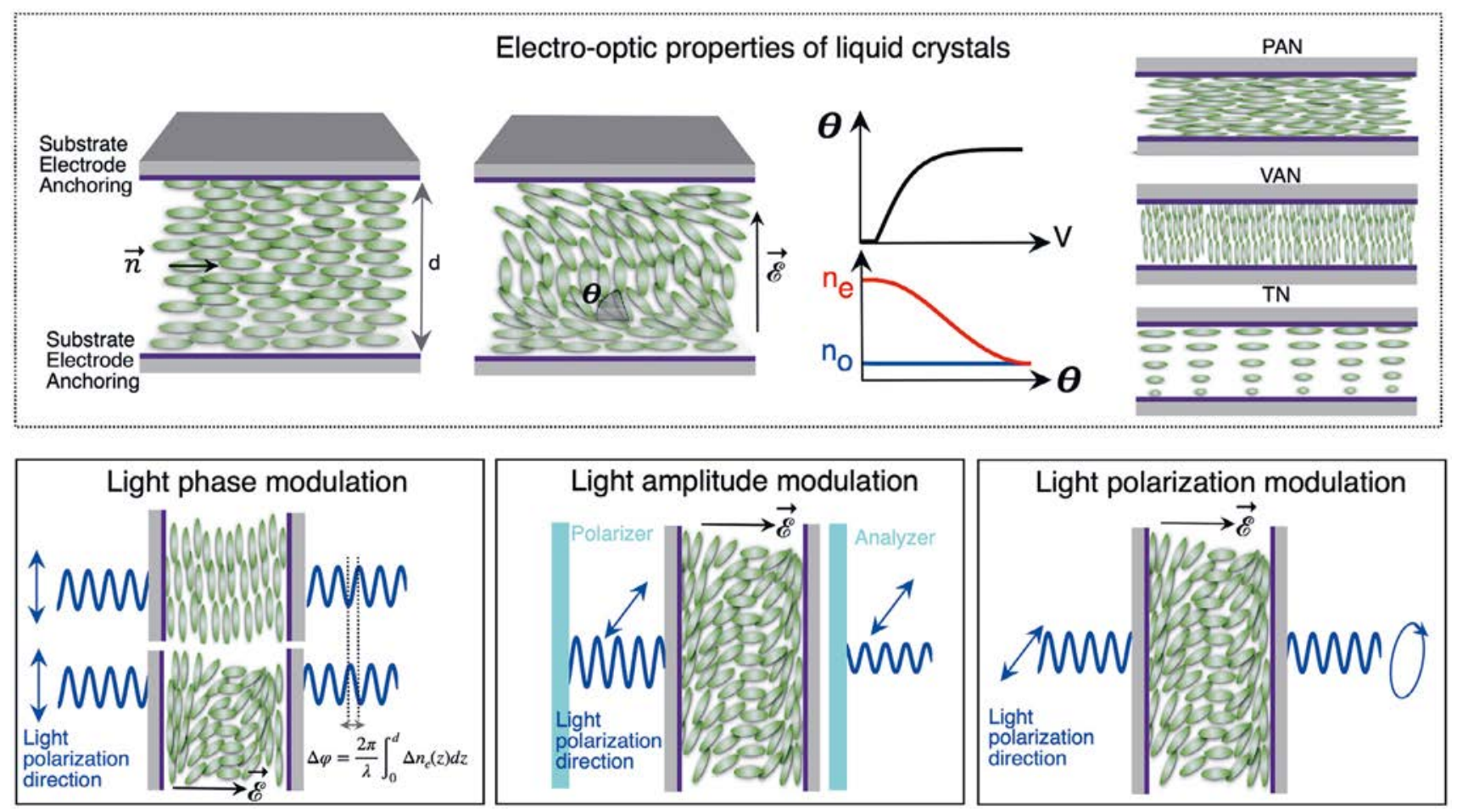

the denomination most often refers to non-mechanical components which exploit the electro-optical anisotropy of liquid crystals (LCs). Thereafter, we will focus onto the physical and technical characteristics of the LC-based SLMs (LC-SLMs) [1].

\section{PRINCIPLE AND APPLICATIONS}

Principle. Liquid crystals are organic materials whose physico-chemical properties are intermediate between those of solids and liquids. The elongated LC molecules therefore have both a structural order and anisotropy specific to crystals such as optical, dielectric or even elastic anisotropy. The optical anisotropy is generally higher than in crystals: an optical birefringence ranging between 0.1 and 0.2 for example is common in LCs. The existence of a liquid order also guarantees the fluid nature of the different LC mesophases and, to some extent, the tunability of their properties. These characteristics, combined with a wide spectral transparency, have made LCs materials widely used in optics.

In the nematic mesophase, molecules do not have a positional order but are oriented in a preferential
Figure 1: Electro-optical properties of nematic liquid crystal layers enable to locally change the phase of the propagating readout light. The application of the electric field induces an average molecular rotation, which in return changes the refractive index according to the input light polarization. Typical planar anchoring conditions can be vertical (VAN) or horizontal (PAN) or both, e.g. twisted (TN). Such a simple device allows for the modulation of the phase, amplitude or polarization of light according to the design details and the presence or absence of additional polarizing elements.

direction, defined by a vector $\overrightarrow{\boldsymbol{n}}$, the so-called director axis. They feature properties of an anisotropic uniaxial medium with an optical axis oriented along $\overrightarrow{\boldsymbol{n}}$. This direction can be experimentally specified by defining specific boundary conditions at the surface of the sample. Typical anchoring conditions can be planar (PAN), homeotropic (VAN) or twisted (TN). Furthermore, the orientation of the director axis can be controlled by applying external electric and / or magnetic fields. As a matter of fact, the application of an electric field results in the creation of elastic forces leading to the reorientation of the molecules that tend to line up in the direction where the strain energy is minimal. For LCs with a positive dielectric anisotropy, the minimum energy is reached when the molecules are aligned in the direction of the electric field. The strength of the electric field modulates the average molecular orientation. Therefore, the optical refractive index of the medium is electrically-controlled and the phase of a propagating light is modified accordingly.

Spatial control of the applied electric field, on one or two dimensions, offers the ability to spatially modulate the phase of an incident optical wave. The latter is referred in the following as the "readout beam", while the recording signal contains the information to be "printed" on the phase of the readout light. The readout beam has to be polarized. In addition, its polarization is a mean to control the parameter modulated by the LC component, whether it is the phase, amplitude or polarization [2]. Indeed, projection of an initially linearly polarized light at $45^{\circ}$ with respect to the LC extraordinary axis provides a phase-mismatch between the two crossed-polarized components. This variable phase 
shift allows the modulation of the light amplitude if the component is placed between polarizer and analyzer, and the modification of the linear polarization state to elliptical otherwise. Subsequently, phase-only, amplitude-only, polarization, or the combination of phase-amplitude modulation can be readily realized with a LC-SLM, as illustrated in Figure 1.

Applications. For decades, the large market of image projection and displays has fed the development of LC-SLMs that address otherwise uncountable applications in a wide field of scientific investigations. Nowadays, SLMs are used in fields as varied as imaging, digital holography, optical switching, microstructure fabrication, optical vortex generation. In the context of adaptive optics, SLMs are employed to correct the wavefront of lasers and optimize the point spread function for biomedical applications and microscopy. In addition, SLMs enable ultrashort optical pulse shaping through a process known as Fourierdomain pulse shaping. Recently, such devices have also been used in the field of telecommunications in order to achieve modal multiplexing in multimode optical fibers. Some illustrations are available in Figure 2.

\section{SLM MAJOR FAMILIES}

\section{AND PERFORMANCES}

Most of commercial SLMs are electrically-addressed, for instance through standard digital video interface with each grey level being related to a given voltage. They can operate either in reflection or in transmission.

Transmissive SLMs. Transmissive SLMs include a nematic LC layer confined between two transparent conductive windows. At least one electrode is segmented, in order to provide individual electrical control over a certain amount of pixels. A particular type of transmissive SLM is the well-known LCD (Liquid Crystal Display), for amplitude light modulation. LCD relies on TN-type LC-SLM, placed between parallel or crossed polarizers.

Reflective SLMs. Reflective SLMs are particularly sought as they enable to fold the associated optical system, while light propagates twice in the modulating layer, which, in turn, increases the dynamic range. In this family, the most popular technology is LCoS (Fig. 3): Liquid Crystal on Silicium, mainly used for phase-only or amplitudeonly light modulation. Most of current commercial electrically-addressed LCSLMs are based on this technology.

\section{Therefore, the nature and concentration of solutes in liquid water is the main contrast factor for biological systems.}

LCoS are microdisplays, composed of a layer of nematic liquid crystals enclosed between a transparent electrode and a matrix of CMOS (complementary metal oxide semiconductor) integrated circuitry on a silicon backplane. The latter operate in reflection through a reflective treatment deposited on the CMOS matrix. Anchoring layers on one side of the electrode and on the reflective layer allow the molecules to be oriented in a direction parallel to the surface. An electric field maintained between the transparent electrode and the semiconductor controls the local average molecular orientation of the liquid crystal and modulates its refractive index.

Performances criteria. The different components of the LC-SLM multi-layer structure can be individually optimized according to the targeted application. Performances of

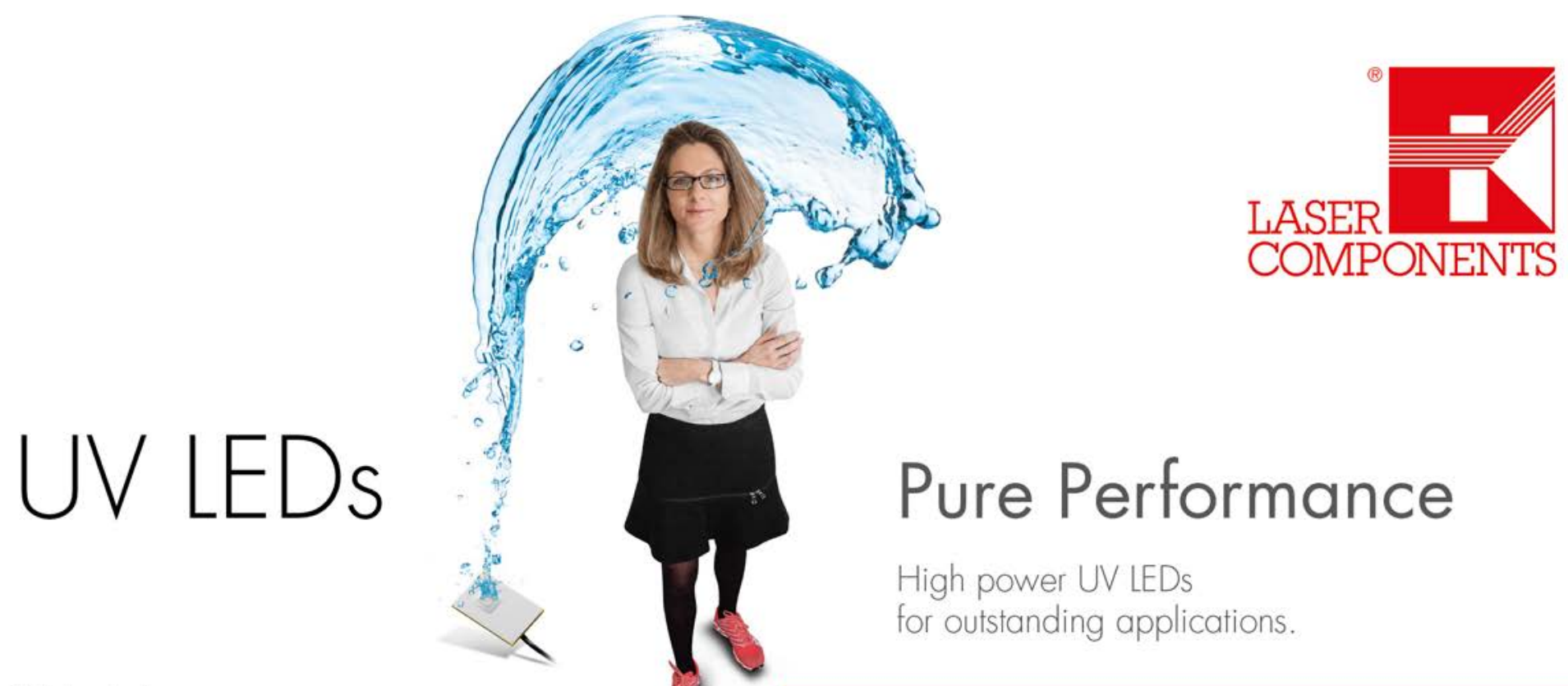


LC-SLMs are then characterized as $a$ priority by their active area, transmittance / reflectance, spectral acceptance, spatial resolution, response time and modulation dynamics.

Modulation range and response time. The modulation range is the maximum retardation that can be applied to a given wavelength while the dynamic response time is defined as the switching time from $10 \%$ to $90 \%$ and from $90 \%$ to $10 \%$ (rise and fall time). Theses two features are primarily determined by the LC layer material and thickness. Independently from the technology, the thickness of the LC layer is generally limited to $20 \mu \mathrm{m}$ in most SLMs, and results from a balance between the desired modulation range (e.g. maximum phase modulation), maximum control voltage, molecular disorder issues, and dynamic response time. For radiation in the visible spectral range, the phase modulation evolves between 0 and $2 \pi$ or 0 and $4 \pi$. The dynamic response time, meanwhile, ranges typically between 1-100 ms for $10-90 \%$ rise and fall times.
Active area. LC-SLMs for scientific applications present an active area usually around $1-2 \mathrm{~cm}^{2}$, with some specific extension in the array configuration, up to $7 \mathrm{~cm} \times 1 \mathrm{~cm}$.

Spatial resolution. The spatial resolution is related to two parameters: the pixel density and the cross-talk between adjacent pixels. The pixel pitch depends on the category of SLMs. Higher pixel densities are achieved with LCoS, typically $1920 \times$ 1080 pixels, but also up to $4160 \times 2464$. Transmissive SLMs are restricted to a larger pixel size (a few tens of $\mu \mathrm{m}$ for transmissive SLMs, as opposed to a few $\mu \mathrm{m}$ for LCoS). When specified beyond the simple number of pixels, the spatial resolution of an LC-SLM is around 40 lines per $/ \mathrm{mm}$, that is between $20-30 \mu \mathrm{m}$.

Filling factor. The electrically controlled LC-SLM makes it possible to control the properties of the readout light over a limited number of zones predefined by the manufacturer, this number being approximately equal
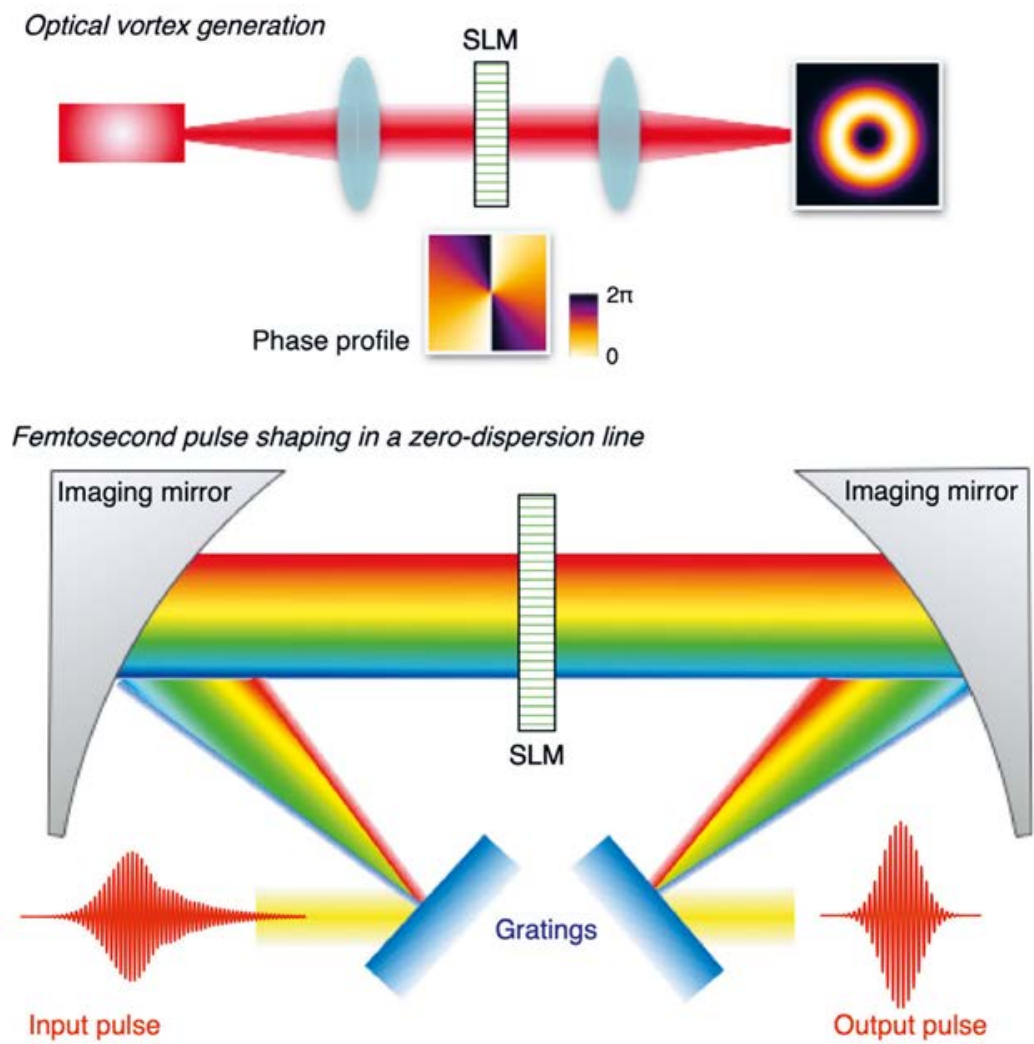

Figure 2: Illustration of some applications of SLMS. Top: a spiral phase pattern leads to optical vortex beams [3]. Bottom: a SLM inserted in a zerodispersion line enables the temporal shaping of a femtosecond pulse [4].

Spectralacceptance. CommercialSLMs make it possible to address different spectral ranges, with bandwidth around $200 \mathrm{~nm}$, centered in the visible, nearinfrared or close to telecommunication bandwidths. A remaining drawback of the electrically-addressed LC-SLM technology is the presence of a top electrode. Most of the time, Indium Tin Oxyde (ITO, typically transparent over $0.3-1 \mu \mathrm{m}$, with partial transmission up to $1.5 \mu \mathrm{m}$ ) is employed, which tends to reduce the spectralacceptance, by comparison with the LC mixture itself. Moreover, as the phase modulation range scales with the optical frequency, extending the spectral range towards the infrared tends to require thicker LC layers, which, in turn, constraints the electrical addressing scheme. These two features limit the practical use ofLC-SLMs in the visible and near infrared spectral range.

to the number of electrode segments. has discontinuities in the modulated optical property. Inactive gaps are

Reflectivity (transmittance). The reflectivity (transmittance) is not $100 \%$ into higher orders due to the grating some part of light is also scattered and absorbed at the interpixel gaps. In addition, the overall reflectivity (transmittance) is limited by losses at the structure, the electrode transparency, lectric coating in LCoS. Typical values spread between $70 \%$ and $90 \%$. 
Figure 3: Structure and cross-section of an LCOS SLM and photograph of an industrial product from Holoeye.

Flicker. The flickering phase corresponds to the phase fluctuation due to electric polarization of the LC molecules and can be reduced to $0.01 \pi$ by carefully designing control electronics.

Damage threshold. LC-SLM can tailor the properties of high power laser beams. Some damages might alter the SLM behavior, either due to laser ablation of one of the LC confining substrates, or to heating of the LC layer. According to the available data, the damage threshold is limited by electrodes and/or metallic coating in LCoS and is around 5W/ $\mathrm{cm}^{2}$ for continuous light radiation, and decreases to $0.1 \mathrm{~J} / \mathrm{cm}^{2}$ for pulsed femtosecond lasers.

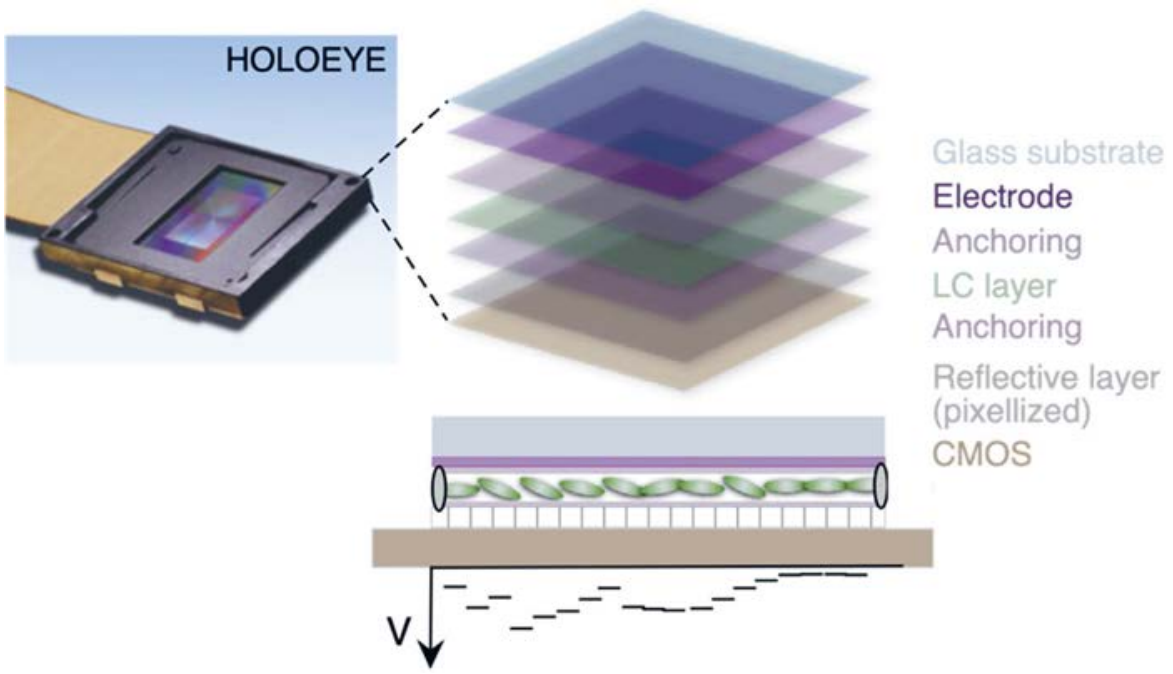

\section{LIMITATIONS}

\section{AND RECENT ADVANCES}

Although LC-SLMs are very performant and popular optical systems, some limitations can be deduced from the performances detailed above. Among them, the pixelisation and limited spectral acceptance in the mid-infrared spectral range are the most challenging issues. Nevertheless, research and development of innovative LC-SLMs is still very active. In particular, other solutions for controlling the birefringence are being investigated.

Replacing the electrical addressing by an optical addressing solves the pixelisation issue and ensures arbitrary and continuous phase modulation.
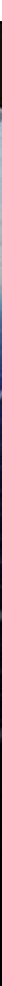

\section{TRL9 \\ Specialty Fibers \\ - Rad Hard Space \\ Grade Doped Fibers \\ - Space Grade PM \\ Gyro Fibers}

TRL9

$\mathrm{LiNbO}_{3}$ modulators

Amplitude \& Phase

$\mathrm{LiNbO}_{3}$ Modulators

Custom $\mathrm{LiNbO}_{3}$

Modulators

\section{iXblue}


In so-called light valve modulators, or OASLM, a biased photo-conductive substrate replaces the segmented electrode and the voltage across the LC layer is locally controlled by an ancillary absorbed control beam, often referred as the "recording" beam, as opposed to the "readout" beam. This electrode is, however, mandatory, as an oscillating electric field has to be maintained across the LC layer to control the average orientation of the molecular director. Moreover, conventional optical valves require an isolation layer to prevent crosstalk between the recording and readout beams.

Finally, novel technological developments in this field are steadily proposed. Thermal or thermooptical control of the LC layer or photo-polymerization of the anchoring layer might be promising methods to provide continuous phase modulation while eliminating the need for an electrode. Dielectric metasurfaces might also be part of the next-generation of SLMs.

\section{CONCLUSION}

Spatial light modulators, thanks to their dynamic attractive optical capacities and to their technological maturity, are widespread in several scientific and industrial domains. The most common components exploit the electro-optical anisotropy of liquid crystals and are commercialized following LCD or
LCoS technologies. Several performance criteria, such as panel resolution, active optical area, refreshing rate and spectral acceptance must be considered in order to define the most appropriate SLM for a targeted application. It should be noted that several developments towards innovating technologies could further boost the practical applications in the coming years.

\section{REFERENCES}

[1] Spatial Light Modulator Technology: Materials, Devices, and Applications, ed. Efron (1995)

[2] C. Rosales-Guzmán and Andrew Forbes, How to Shape Light with Spatial Light Modulators, SPIE Spotlight (2017)

[3] A. A. Zinchik, Application of spatial light modulators for generation of laser beams with a spiral phase distribution, Sci. Tech. J. Inf. Technol., Mech. Opt. (2015)

[4] A. M. Weiner, Femtosecond pulse shaping using spatial light modulators, Rev. Sci. Instrum. 71, 1929-1960 (2000)

[5] N. Collings et al., The Applications and Technology of Phase-Only Liquid Crystal on Silicon Devices, J. Disp. Technol. 7, 112-119 (2011)

\begin{tabular}{|c|c|c|c|}
\hline MAKES & SUPPLIER & PRODUCTS & CONTACT \\
\hline Hamamatsu & Hamamatsu Photonics France SARL & LCoS & $\begin{array}{l}\text { info@hamamatsu.fr } \\
\text { +33 (0)1 } 69537100\end{array}$ \\
\hline Jenoptik & Jenoptik & LCos, trans. SLMs & $\begin{array}{l}\text { produkte@jenoptik.com } \\
\text { +49364165-4243 }\end{array}$ \\
\hline Biophotonics Solution & Biophotonics Headquarters & LCoS & $\begin{array}{l}\text { info@bsifemto.com } \\
+1-508-506-2941\end{array}$ \\
\hline Meadowlark Optics & Photon Lines & LCoS, trans. SLMs & $\begin{array}{l}\text { ag-robert@photonlines.com } \\
\text { +33(0)130089900 }\end{array}$ \\
\hline Thorlabs & Thorlabs SAS France & $\mathrm{LCOS}$ & $\begin{array}{l}\text { Sales.fr@thorlabs.com } \\
\text { +33 (0) } 970444844\end{array}$ \\
\hline Holoeye Photonics AG & Optoprim & LCoS, trans. SLMs & $\begin{array}{l}\text { yjoly@optoprim.com } \\
\text { +33(0) } 141906180\end{array}$ \\
\hline Santec & Santec & $\mathrm{LCOS}$ & $\begin{array}{l}\text { www.santec.com/en/inquiry } \\
+1-201-488-5505\end{array}$ \\
\hline Laser Components & Laser Components S.A.S France & LCoS, trans. SLMs & $\begin{array}{l}\text { info@lasercomponents.fr } \\
\text { +33139595225 }\end{array}$ \\
\hline
\end{tabular}

